\title{
Gemcitabine-loaded liposomes: rationale, potentialities and future perspectives
}

\author{
This article was published in the following Dove Press journal: \\ International Journal of Nanomedicine \\ 31 October 2012 \\ Number of times this article has been viewed
}

\section{Cinzia Federico \\ Valeria M Morittu \\ Domenico Britti \\ Elena Trapasso \\ Donato Cosco}

Department of Health Sciences, Building of BioSciences, University "Magna Græcia" of Catanzaro, Campus Universitario "S Venuta", Germaneto, Italy
Correspondence: Donato Cosco Department of Health Sciences, Building of BioSciences, University "Magna Græcia" of Catanzaro, Campus Universitario "S Venuta", Viale Europa, I-88I00 Germaneto, Italy

$\mathrm{Tel}+3909613694119$

Fax +39096 I369 4237

Email donatocosco@unicz.it
Abstract: This review describes the strategies used in recent years to improve the biopharmaceutical properties of gemcitabine, a nucleoside analog deoxycytidine antimetabolite characterized by activity against many kinds of tumors, by means of liposomal devices. The main limitation of using this active compound is the rapid inactivation of deoxycytidine deaminase following administration in vivo. Consequently, different strategies based on its encapsulation/complexation in innovative vesicular colloidal carriers have been investigated, with interesting results in terms of increased pharmacological activity, plasma half-life, and tumor localization, in addition to decreased side effects. This review focuses on the specific approaches used, based on the encapsulation of gemcitabine in liposomes, with particular attention to the results obtained during the last 5 years. These approaches represent a valid starting point in the attempt to obtain a novel, commercializable drug formulation as already achieved for liposomal doxorubicin $\left(\right.$ Doxil $^{\circledR}$, Caelyx $^{\circledR}$ ).

Keywords: gemcitabine, liposomes, multidrug, poly(ethylene glycol), tumors

\section{Introduction}

The poor efficacy of anticancer drugs is often related to their poor selectivity towards tumor tissue and to their toxicity. Use of innovative drug delivery systems can optimize their therapeutic features, protecting the drug against metabolic inactivation, increasing its plasma half-life, and improving both the therapeutic index and the anticancer efficacy of the drug. ${ }^{1-3}$

Gemcitabine is one of the most widely used anticancer drugs in the treatment of several types of solid tumors, including pancreatic cancer, non-small cell lung cancer, breast cancer, head and neck squamous cell carcinoma, and cervical, bladder, ovarian, and thyroid cancers. ${ }^{4,5}$ Chemically, gemcitabine $\left(2^{\prime}, 2^{\prime}\right.$-difluoro-deoxycytidine, $\left.\mathrm{dFdC}\right)$ is an analog of deoxycytidine, in particular of cytosine arabinoside, from which it differs by two fluorine atoms in position 2 of the deoxyribose (Figure 1). It shows more distinctive pharmacological properties and has a wider spectrum of antitumoral activity than cytosine arabinoside. ${ }^{6}$ Moreover, like cytosine arabinoside, gemcitabine is a prodrug requiring cellular uptake and intracellular phosphorylation.

Gemcitabine passes through the cell membrane with difficulty because it is moved across by passive diffusion, but requires active transporters. Five nucleoside transporters are involved in its uptake, ie, two equilibrative sodium-independent type (hENT1, hENT2) nucleoside transporters and three concentrative sodium-dependent nucleoside type (hCNT1, hCNT2, hCNT3) nucleoside transporters. ${ }^{7,8}$ 


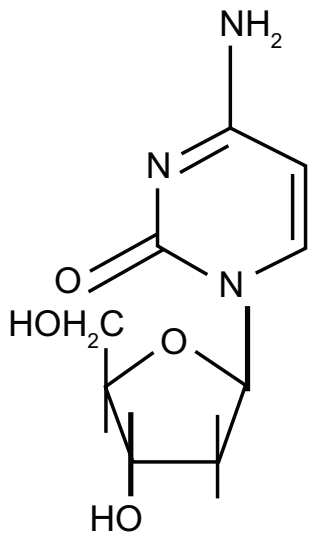

Deoxycytidine

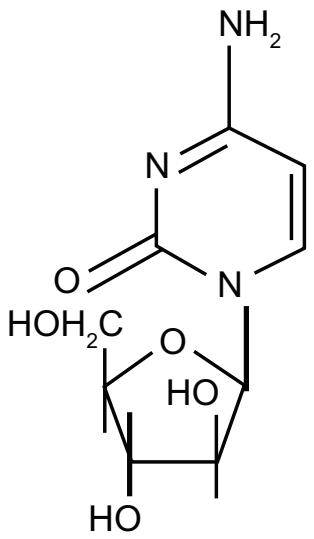

Cytosine arabinoside

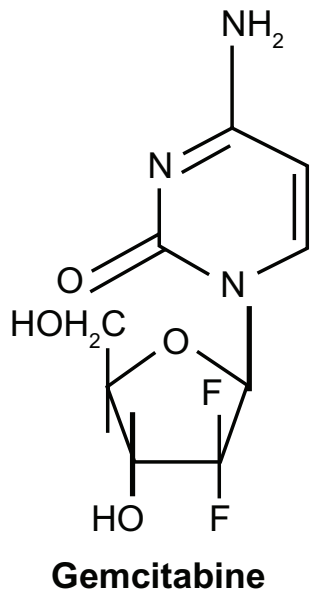

Figure I Structures of deoxycytidine, cytosine arabinoside, and gemcitabine. ${ }^{6}$

Activity of the nucleoside transporters is fundamental to the inhibition of cell growth and promotion of the clinical efficacy of the nucleoside analog, because its uptake is mediated by both of them, even if hENT1 nucleoside transporters are overexpressed. ${ }^{8}$ In fact, many studies have shown that a deficiency in or an inhibition of nucleoside transporters induces considerable resistance of tumor cells to gemcitabine. ${ }^{6,9,10}$

After cellular internalization, the drug is phosphorylated by deoxycytidine kinase into nucleotide monophosphate (dFdCMP) which is then phosphorylated two more times in order to form the triphosphorylated derivative which plays an important role in anticancer drug activity. In particular, gemcitabine diphosphate ( $\mathrm{dFdCDP}$ ) inhibits ribonucleotide reductase, resulting in depletion of the deoxyribonucleotide pool useful in DNA synthesis. ${ }^{11}$ Gemcitabine triphosphate (dFdCTP) binds DNA polymerase, thus competing with the natural substrate (deoxycytidine triphosphate) and causing termination of the DNA helix as a consequence of binding of another nucleotide, ie, steric hindrance due to fluoride. ${ }^{12,13}$ The terminal complex dFdCTP/DNA induces apoptosis in many cancer cell lines because it is not recognized by normal cell repair factors; moreover, gemcitabine is a phase-specific drug because it acts between the $G_{1}$ and $S$ phases of the cell cycle, thus precluding cell proliferation. ${ }^{6}$

Development of resistance to chemotherapeutic compounds compromises their pharmacological efficacy. In addition to being due to decreased numbers or activity of nucleoside transporters, resistance to gemcitabine could be caused by concentration-dependent pumps active on the bilayer of many types of cancer cells which drive the drug out when it reaches certain levels in the cytosol. ${ }^{10}$ In the case of water-soluble drugs, such as gemcitabine, these structures (P-glycoproteins, a product of $m d r l$ gene) do not confer resistance, although the exact mechanism of this phenomenon has not yet been clarified. ${ }^{14}$

The accepted resistance mechanisms are related to: overexpression of cytidine deaminase, an enzyme which is widely distributed in many body compartments and inactivates gemcitabine following administration in vivo, ${ }^{15}$ thereby reducing its antitumoral efficacy; ${ }^{8}$ a deficiency of deoxycytidine kinase, an enzyme involved in gemcitabine metabolism; ${ }^{16}$ and amplification of genes coding for specific enzymes involved in purine and pyrimidine biosynthesis, ${ }^{14}$ in addition to deregulation of p53 which prevents cell apoptosis. ${ }^{6,16,17}$

More specifically, following systemic administration, gemcitabine is quickly metabolized by cytidine deaminase into the inactive compound, $2^{\prime}$-deoxy-2,2'-difluorouridine, and is then cleared by the kidneys. The metabolite is rapidly excreted in the urine, resulting in a half-life of 8-17 minutes, so repeated administration of the drug is necessary in order to assure an adequate pharmacological effect. ${ }^{18}$ Although gemcitabine has a better therapeutic index than some other anticancer drugs, serious side effects, such as myelosuppression, mild and transient neutropenia, thrombocytopenia, and anemia, have been observed during clinical trials. ${ }^{19}$

Due to its complex pharmacodynamic profile, the antitumoral effects of gemcitabine are more dependent on the frequency of administration than dosage, and a suitable therapeutic response can only be obtained following daily administration or prolonged infusion for some types of cancer. ${ }^{20}$ Several strategies have been proposed to obtain efficient delivery of gemcitabine ${ }^{20,21}$ and to reduce the abovementioned therapeutic complications, in addition to improving its anticancer efficacy by circumventing resistance phenomena arising from saturation of efflux pumps and overexpression of enzymes associated with resistance. 
Gemcitabine has been encapsulated in different colloidal devices, eg, liposomes and polymeric nanoparticles, and conjugated with lipophilic compounds in order to develop novel formulations able to improve its biopharmaceutical features. This review describe advances in gemcitabine delivery made possible by the liposomal devices developed in recent years, which have led to the potential for marketing of one or more formulations, as for liposomal doxorubicin $\left(\right.$ Caelyx ${ }^{\circledR}$, Doxil $\left.^{\circledR}\right) .{ }^{22}$

\section{Liposomal delivery of gemcitabine}

Liposomes are biocompatible and biodegradable selfassembled vesicles, characterized by a supramolecular lipidic organization which is the same as that found in the natural membranes of living cells (Figure 2). ${ }^{23}$ This is an advantage from the standpoint of biocompatibility and biodegradability because it induces neither side effects nor accumulation. Of all the possible nanomedicine platforms, liposomal formulations are the ones that have been approved by the US Food and Drug Administration for the treatment of cancer. ${ }^{24,25}$ It has been well demonstrated that use of liposomes in the treatment of solid tumors in particular protects the incorporated molecule from being inactivated following intravenous administration, which reduces accumulation of the anticancer drug in healthy tissues before it reaches the desired site of action. ${ }^{23}$ Consequently, liposomes enable a decrease in nonspecific toxicity and an increase in concentration of the encapsulated drug in specific body compartments. ${ }^{2}$ Their structure allows them to encapsulate both hydrophilic and hydrophobic drugs. The size of the liposome depends on its composition and the preparation method used, and influences drug-loading capacity. ${ }^{26}$

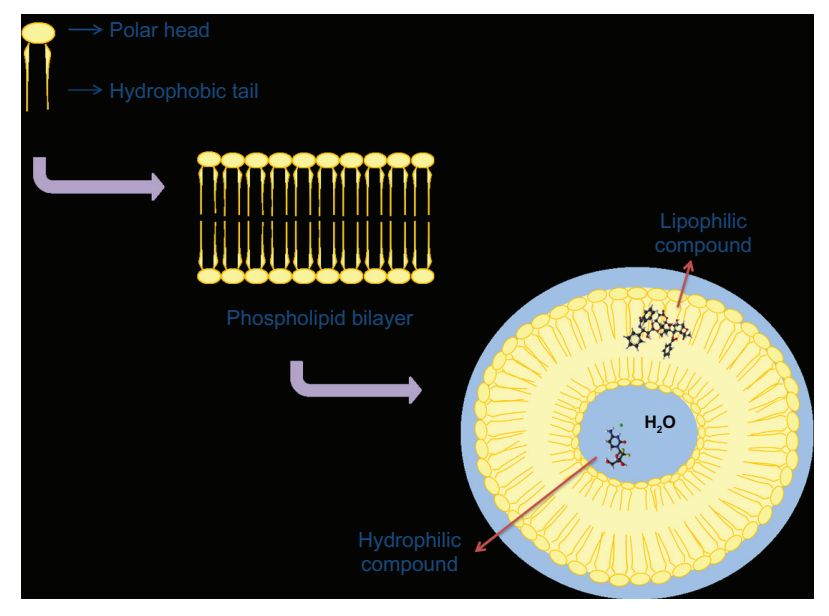

Figure 2 Schematic representation of a liposomal structure with a characteristic microenvironment and possible drug encapsulation as a function of its physicochemical features.
A potential problem related to the intravenous administration of liposomes is that they may be absorbed by macrophages in the reticuloendothelial system as a consequence of their opsonization as foreign molecules, ie, interaction with plasma proteins and lipoproteins, leading to destruction of these vesicles. ${ }^{2,27}$ This phenomenon can modify the biodistribution and pharmacokinetic features of liposomes and consequently those of the encapsulated anticancer drug, leading to a lack of therapeutic accumulation in tumor tissue and, in many cases, with a considerable degree of systemic toxicity. ${ }^{28}$ This phenomenon ${ }^{13,29}$ could be avoided by decreasing the mean vesicle size using a variety of methods, such as extrusion through polycarbonate filters, sonication techniques, or modulation of their surface charge by coating with a polymer such as poly(ethylene glycol) (PEG). PEG is a polymer which has been approved by the Food and Drug Administration as a constituent of pharmaceutical preparations for systemic administration because it is nontoxic and biocompatible. ${ }^{30}$ PEG also prevents interaction with plasma proteins ${ }^{31}$ as a result of the presence of bulky groups on the hydrophilic polymer, which prevent or reduce electrostatic and hydrophobic interactions, which are the basis for adsorption of plasma opsonins, resulting in a lower uptake by the reticuloendothelial system. ${ }^{30}$

PEGylated liposomes, also known as "stealth liposomes" because of their long systemic circulation time and small size (between 50-200 nm), tend to accumulate more than conventional liposomes of the same mean diameter in tumor tissues because of the enhanced permeation and retention effect. In general, liposomes with a smaller mean size (50-300 nm) can cross the endothelium more easily and reach the tumor target area, which is usually poorly drained by lymphatic vessels, and so remain in the interstices of the tumor for prolonged periods. ${ }^{32}$

The most widely known formulation based on a natural compound associated with a delivery system currently available is doxorubicin hydrochloride encapsulated in unilamellar PEGylated liposomes (Caelyx, Doxil). This formulation, comprised of soy phosphatidylcholine, cholesterol, and distearoyl-phosphoethanolamine conjugated with PEG2000, has a mean carrier size of about $100 \mathrm{~nm}$, allowing reduction of the most important side effects of the drug, in particular its cardiotoxicity and myelotoxicity. ${ }^{33}$ Today, this formulation is the standard treatment for a number of different types of cancer. ${ }^{34}$ This approach has also been useful in investigating the advantages deriving from encapsulation of gemcitabine in a vesicular colloidal device. 
In a manner analogous to the method used to entrap doxorubicin efficiently in liposomal vesicles, a $\mathrm{pH}$ gradient was created in the aqueous compartments of vesicles in order to increase the amount of encapsulated gemcitabine. ${ }^{35,36}$ It has recently been demonstrated that the kinetics of permeation through biomembranes depend on the physicochemical parameters of the antitumoral compound, ie, protonation or deprotonation. ${ }^{37}$ Pretreatment with an ammonium sulfate solution gives rise to the appearance of a gel-like precipitate of the drug within the liposomes, a phenomenon specifically related to protonation of the amino group of gemcitabine (Figure 3). ${ }^{38}$ During experimentation, this approach allowed a drug-loading capacity of more than $90 \%$, both for the precipitation of the drug as a salt sulfate in the aqueous liposomal compartment and for its considerable degree of interaction with phospholipids in the bilayers. ${ }^{35,37}$

Another technological strategy useful for increasing the amount of gemcitabine inside a colloidal device and for protecting the drug from metabolic uptake was discovered during experimentation. This was the development of lipophilic prodrugs, obtained by linking the 4-amino group of gemcitabine with an acyl chain, such as a valeroyl, heptanoyl, lauroyl, or stearoyl derivative. This innovative strategy increased the lipophilicity of gemcitabine, thus improving its stability. In fact, a lipophilic drug is retained to a greater degree within the lipid bilayer as compared with its hydrophilic analog, which has a tendency to spread rapidly through the bilayer. ${ }^{15}$ Furthermore, the process of acylation protects gemcitabine from cytidine deaminase by reducing its catabolism in plasma, thus enhancing the pharmacological activity of the active compound in comparison with the drug administered in its free form. ${ }^{15,39}$

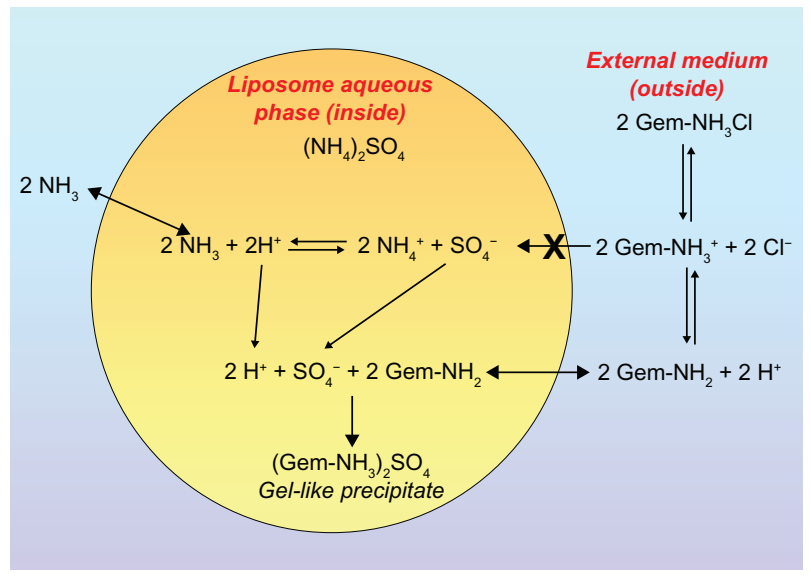

Figure 3 Schematic representation of the gemcitabine encapsulation process in liposomes using a pH gradient elicited by coencapsulation of a $250 \mathrm{mM}$ ammonium sulfate solution. (2006 Oxford University Press. Reproduced with permission from Celano M, Calvagno MG, Bulotta S, et al. Cytotoxic effects of gemcitabine-loaded liposomes in human anaplastic thyroid carcinoma cells. BMC Cancer. 2004;4:63. ${ }^{35}$
This strategy was also used by Couvreur et al, who manufactured a squalenoyl-gemcitabine derivative by coupling the drug with $1,1^{\prime}, 2$-tris-nor-squalenic acid to obtain a novel anticancer nanocompound known as 4-(N)-tris-nor-squalenoylgemcitabine, which is able to autoassemble in nanoaggregates organized within an inverse hexagonal structure..$^{40}$

This new nanomedicine was able to bypass different types of resistance mechanisms, resulting in a greater degree of tumoral cytotoxicity in comparison with free gemcitabine. ${ }^{40}$ Squalenoyl nanoaggregates can also penetrate into cells in the absence of hENT1 transporters, probably via endocytosis or by diffusion or insertion of the squalenoyl molecules through or into the cell membrane. Moreover, squalenoyl-gemcitabine nanoaggregates overcame the resistance to deoxycytidine kinase in the cytoplasm because they maintained slow intracellular release of the drug with a low level of intracellular deoxycytidine kinase activity; as long as the gemcitabine remained covalently bound to the squalene via its amino group, it also showed good resistance to deamination by intracellular deaminases. In addition, the nanoaggregates demonstrated a greater ability to induce S-phase arrest in cancer cells and their apoptosis, probably as a consequence of greater and more prolonged cellular retention of the drug. ${ }^{41}$

Furthermore, squalenoylation caused slower metabolism of gemcitabine in plasma because the nanoassemblies act as a prodrug, resulting in intracellular release of the drug after cleavage by plasma proteases (cathepsin B and D) which have a key role in the degradation of amide-bearing drugs. ${ }^{40}$ The same prodrug was used to increase the encapsulation of gemcitabine within PEGylated liposomes. The efficiency of drug incorporation was confirmed by differential scanning calorimetry and small-angle $\mathrm{x}$-ray scattering analysis. ${ }^{42}$

As previously reported, the presence of hydrophilic polymers on the surfaces of liposomes is important for preventing their uptake by the reticuloendothelial system and consequently for increasing the half-lives of colloidal devices and their prolonged presence in the bloodstream. Recently, a phenomenon known as accelerated blood clearance has been observed following repeated administration of PEGylated liposomes. ${ }^{43}$ Accelerated blood clearance seems to be due to increased synthesis of anti-PEG IgM by the spleen, as a consequence of protracted injections of the formulation, and selectively binding of PEG, leading to rapid elimination of the carrier from the bloodstream via accumulation in the liver and spleen. ${ }^{43-45}$ Reduction of the blood circulation time of the colloid can alter its pharmacokinetic properties and consequently cause side effects related to drug-loaded 
liposomes. ${ }^{45,46}$ This limits the reproducibility of the pharmacokinetic parameters of a PEGylated carrier so, in order to avoid this side effect, some modifications were made to the surface of the colloidal device by binding polymers such as N-vinyl-2-pyrrolidone, poly(4-acryloylmorpholine), or poly(N,N-dimethylacrylamide). ${ }^{47,48}$

A further strategy for improving the biopharmaceutical properties of gemcitabine by rendering it resistant to the abovementioned phenomena was to conjugate a PEGgemcitabine with a folate moiety, the latter being chosen as a targeting agent because its receptor is overexpressed in many tumors. This was done with the aim of obtaining increased localization of the nucleoside analog in the tumor, ${ }^{49}$ and this bioconjugation allows $\mathrm{pH}$-dependent release of the drug independent of the enzyme process (Figure 4). Among all these techniques, encapsulation of gemcitabine in a liposomal carrier allows the easiest modulation of its biopharmaceutical properties with no variation in its chemical structure. ${ }^{50}$

\section{In vitro antitumoral activity of gemcitabine-loaded liposomes}

Liposomes can improve the in vitro antitumoral activity of gemcitabine as a consequence of the lipid composition of the colloidal formulation. ${ }^{20,36}$ In the last few decades, many studies have been performed in order to identify a suitable lipid composition for systemic administration of anticancer drugs, especially for the treatment of solid tumors. Physicochemical and technological characterization of different liposomal formulations showed that a mixture of distearoyl phosphatidylethanolamine (DSPE)-mPEG2000, cholesterol, and dipalmitoyl phosphatidylcholine (DPPC) provided optimal results in terms of gemcitabine delivery. The presence of cholesterol conferred rigidity to the bilayer in addition to providing colloidal stability, ${ }^{2}$ while the PEGylated agent enabled a long circulation time as a result of its shielding effect on the polar heads of DPPC leading to low zeta potential values, a characteristic which influences circulation time in the bloodstream, opsonization, uptake by the reticuloendothelial system, and interaction within the biological compartments. ${ }^{27,36}$

Moreover, gemcitabine did not induce significant changes in the surface charge when it was entrapped in the aforementioned formulation. ${ }^{36}$ On the contrary, gemcitabine was found to be able to interact with the lipids at the level of the polar heads, reducing the fluidity of the lipid bilayer, thus preventing the formation of vesicular aggregates. ${ }^{37}$

Many studies have been performed using different cell lines to assess the activity of gemcitabine-loaded liposomes. Calvagno et al compared the pharmacological and cytotoxic effects of gemcitabine-loaded liposomes with those of the free drug in a colon carcinoma cell line. ${ }^{36}$ This research showed that the PEGylated liposomal formulation had greater cytotoxic activity (evaluated as a function of drug concentration following 48 hours of incubation) than the free form of the drug, probably because encapsulation of the antitumoral compound in a liposomal system enabled greater intracellular uptake due to the ability of the vesicles to penetrate the cell membrane by fusion or endocytotic mechanisms. ${ }^{11,51}$
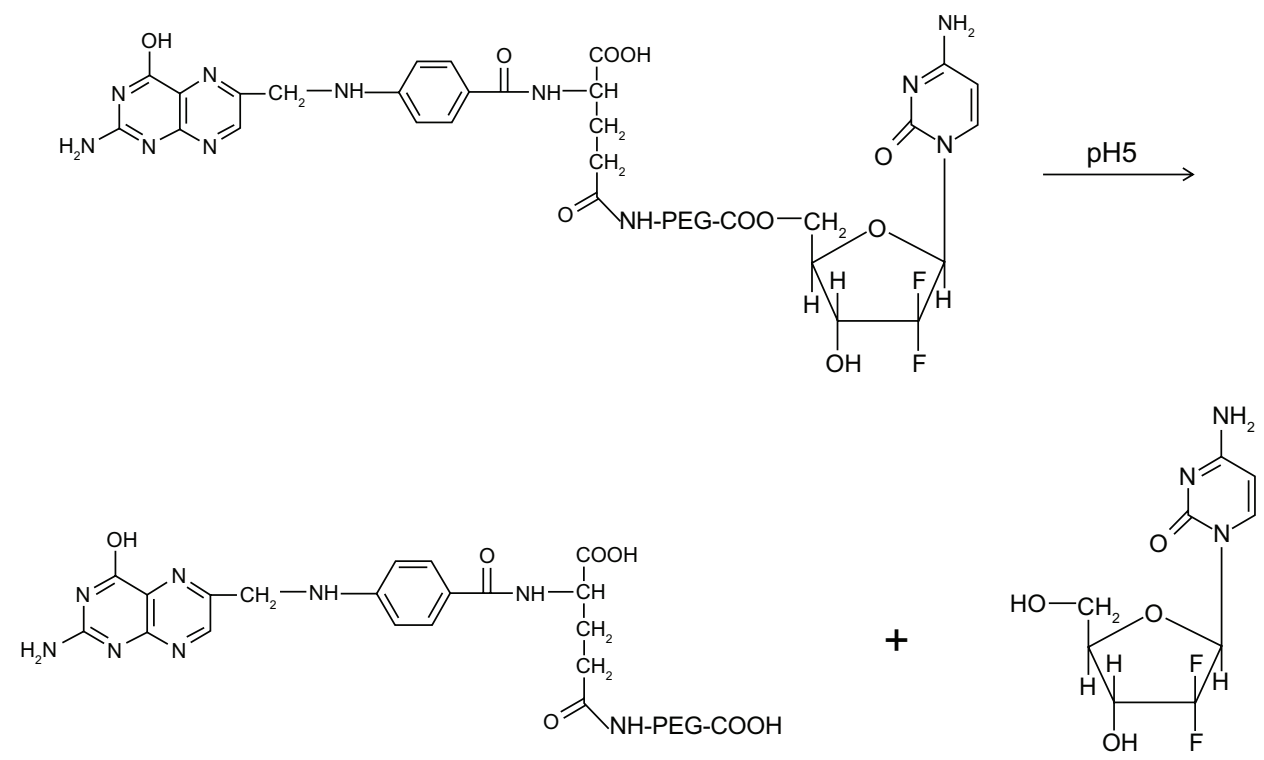

Figure 4 Chemical structure of PEG-folate-gemcitabine macromolecule and process for release of the nucleoside analog as a consequence of $\mathrm{pH}$ variation. Abbreviation: PEG, poly(ethylene glycol). 
Free gemcitabine did not have significant cytotoxic effects on the colon carcinoma cells at any of the experimental drug concentrations, while the liposomal formulation showed high antitumoral efficacy even at low drug concentrations. ${ }^{36}$ Electrostatic charge and the presence of PEG moieties on the liposomal surface were the most important factors influencing interaction between the vesicular structures and the biological membranes. . $2,53^{2}$

Subsequently, the effect of liposomal device size on its interaction with cancer cells was demonstrated. The antitumor activity of gemcitabine-loaded PEGylated unilamellar liposomes was tested in anaplastic thyroid cancer cells in vitro in terms of dose-dependent antitumor effect and incubation time, as shown in Figure 5. The results show that the colloids could significantly improve the cytotoxicity of the drug at a concentration of $1 \mu \mathrm{M}$ after 12 hours of incubation, whereas the free drug only showed significant pharmacological activity after 72 hours of incubation. This trend was confirmed by increasing the duration of exposure of the anaplastic thyroid cancer cells to liposomal gemcitabine during incubation; in this case, the liposomal formulation resulted in $100 \%$ cell mortality at the aforesaid drug concentration after only 24 hours. ${ }^{21,54}$

Similar experiments were performed using other types of thyroid cancer cell lines (TPC-1, B-CPAP) with the aim of obtaining further evidence that the liposomal PEGylated formulation increases cellular uptake of gemcitabine and consequently amplifies its pharmacological effect. ${ }^{51}$ This was confirmed by confocal laser scanning microscopy (CLSM); after 6 hours of incubation with fluorescein-labeled PEGylated liposomes, dissemination of the colloid was observed in all cellular compartments of the B-CPAP cells, including the cell membrane and cytoplasm, thus demonstrating internalization of the colloid (Figure 6).

The same liposomal formulation (containing a 6:3:1 molar ratio of DPPC to cholesterol to DSPEMPEG2000) was proposed for the treatment of multiple myeloma. The growth-inhibiting activity of gemcitabineloaded liposomes as compared with the free drug was assayed in vitro using U266 (autocrine, interleukin-6independent) and INA-6 (interleukin-6-dependent) multiple myeloma cell lines, which are considered to be an in vitro
A
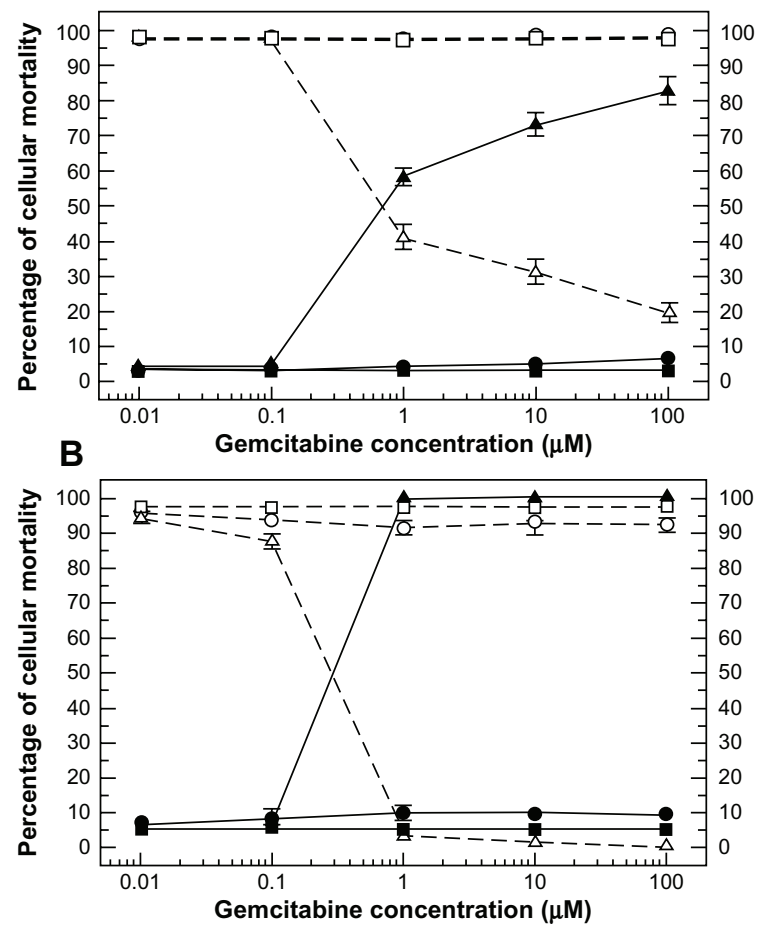

\section{C}
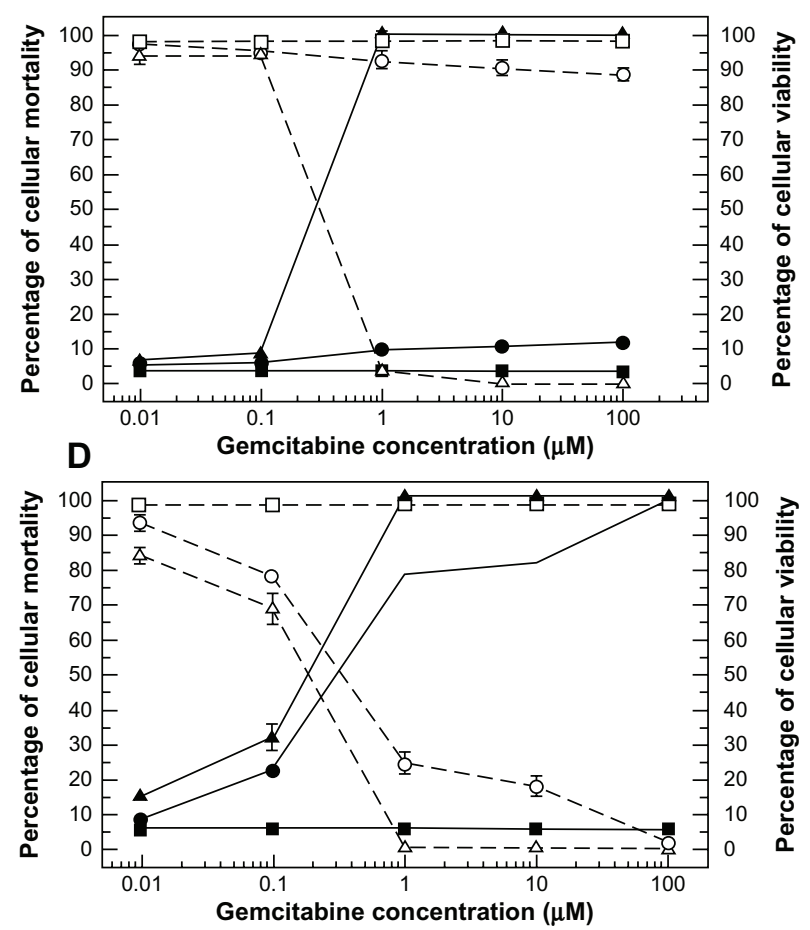

Figure 5 Dose-dependent cytotoxic effect of free gemcitabine (circle) versus gemcitabine-loaded PEGylated unilamellar liposomes (upwards triangle) against anaplastic thyroid carcinoma cells at different exposure times of 12 hours (A), 24 hours (B), 48 hours (C), and 72 hours (D).

Notes: The cytotoxic effect of the drug is expressed both as the percentage cell mortality (filled symbols and solid line) and the percentage cell viability (hollow symbols and dashed line). Cell mortality was evaluated by Trypan blue dye exclusion assay, while cell viability was evaluated by MTT testing. (घ, 口) represents untreated control cells and always shows mortality $\leq 5.5 \%$ and cell viability $\geq 97 \%$. Unloaded liposomes showed similar values to controls (data not reported). Error bars, if not shown, are seen as symbols. Results are presented as the mean \pm standard deviation of five different experiments. (2008 American Scientific Publishers. Reproduced with permission from Celia C, Calvagno MG, Paolino D, et al. Improved in vitro anti-tumoral activity, intracellular uptake and apoptotic induction of gemcitabine-loaded pegylated unilamellar liposomes. J Nanosci Nanotechnol. 2008;8(4):2102-21 13..54 

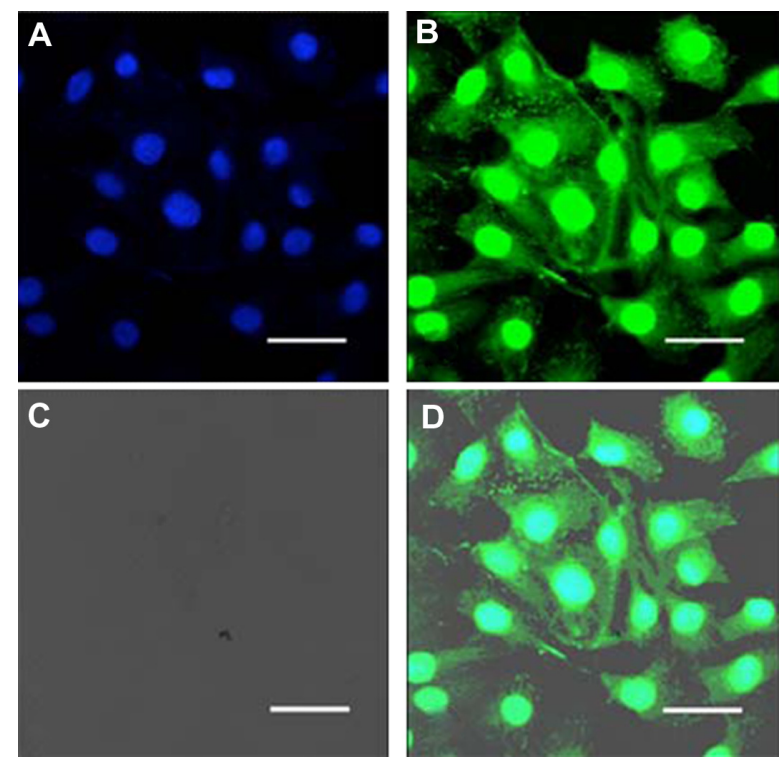

Figure 6 Confocal laser scanning micrographs of B-CPAP cells treated with fluorescein-labeled PEGylated unilamellar liposomes after 6 hours of incubation. (A) Hoechst filter, (B) FITC filter, (C) transmission mode, and (D) overlay.

Note: Bar $30 \mu \mathrm{m}^{5}{ }^{5}$

Abbreviations: FITC, fluorescein isothiocyanate; PEG, poly(ethylene glycol).

model with molecular features similar to those of relapsing multiple myeloma lesions. ${ }^{55}$ The gemcitabine-loaded PEGylated liposomes had better growth-inhibiting activity than the free drug in both cell lines in terms of dosedependent and incubation time effects, leading to enhanced antiproliferative and proapoptotic activity of the antitumoral compound. In addition, the authors demonstrated the ability of the vesicular carriers to penetrate into the cytoplasm, thus delivering the encapsulated gemcitabine directly to its site of action. ${ }^{55}$ A liposomal formulation containing a $0.1 \mu \mathrm{M}$ drug dose induced $99 \%$ inhibition of INA- 6 cell growth after 72 hours of treatment, probably because of massive induction of apoptosis, and complete inhibition of proliferation of INA-6 cells, whereas a 10-fold higher concentration of the free drug was needed to achieve the same effect. This result is very important because it demonstrated inhibition of proliferation at a low drug concentration, which represents a significant advantage in terms of reducing side effects. ${ }^{55}$ Hence, liposomal delivery of gemcitabine consistently and significantly increased induction of apoptosis and achieved complete inhibition of cancer cell proliferation (Figure 7).

Similar results were obtained from in vitro studies using pancreatic tumor cells (BxPC-3 and PSN-1) which showed a significant time-dependent and dose-dependent reduction of cell viability following exposure to liposomal gemcitabine with respect to the free compound. ${ }^{11}$
Experimental CLSM investigations were also performed to evaluate whether the improved cytotoxic effects of gemcitabine-loaded PEGylated liposomes (with respect to conventional drug in saline solution) were related to greater cellular internalization of the active compound mediated by the vesicular carrier. CLSM demonstrated efficient interaction between fluorescein-DHPE-labeled liposomes and the cell membrane in BxPC-3 cells. The experiment was performed at different incubation times $(3,6,12$, and 24 hours), showing that the cell bilayer became stained after just 3 hours of incubation, while significant cytoplasmic localization of the colloid was detected after 24 hours of incubation (Figure 8). ${ }^{11}$

\section{In vivo antitumoral activity of gemcitabine-loaded liposomes}

Many experiments have also been performed using in vivo models to investigate whether entrapment of gemcitabine within liposomal carriers may actually protect the drug from rapid enzymatic degradation, mediate its passive accumulation within tumor tissue via the enhanced permeation and retention effect, and increase its antitumoral activity against several types of cancer. For example, the anticancer effect of gemcitabine-loaded PEGylated liposomes was assessed using two xenograft mouse models of human pancreatic carcinoma, in which BxPC-3 and PSN-1 cells were inoculated into CB-17 SCID mice and the activity of the liposomal formulation was compared to that of the conventional drug in saline solution. It was found that a three-fold lower drug dose in the vesicular formulation had a greater antitumoral therapeutic effect than did the free form of gemcitabine, achieving a significant increase in survival rates for the treated mice. ${ }^{11}$ Pharmacokinetic studies confirmed an increase in the half-life of the drug after its encapsulation in the colloid; liposomal gemcitabine was still detectable 24 hours after administration, while the free drug showed fast clearance from plasma due to rapid enzymatic conversion into the inactive metabolite, $2^{\prime}, 2^{\prime}$-difluorodeoxyuridine. ${ }^{10,14}$

Moreover, this investigation confirmed better protection of the drug from plasma enzymes when it was entrapped in the vesicular carrier, an 8-fold increase in the half-life of the drug in plasma, and effective tumor-targeting activity. The area under the concentration-time curve showed that liposomal gemcitabine had increased stability in plasma and explained the increased bioavailability of the drug with respect to its free form. The highest plasma levels of the inactive metabolite, $2^{\prime} 2^{\prime}$-difluorodeoxyuridine, produced by enzymatic catabolism of gemcitabine, were observed 2 hours 

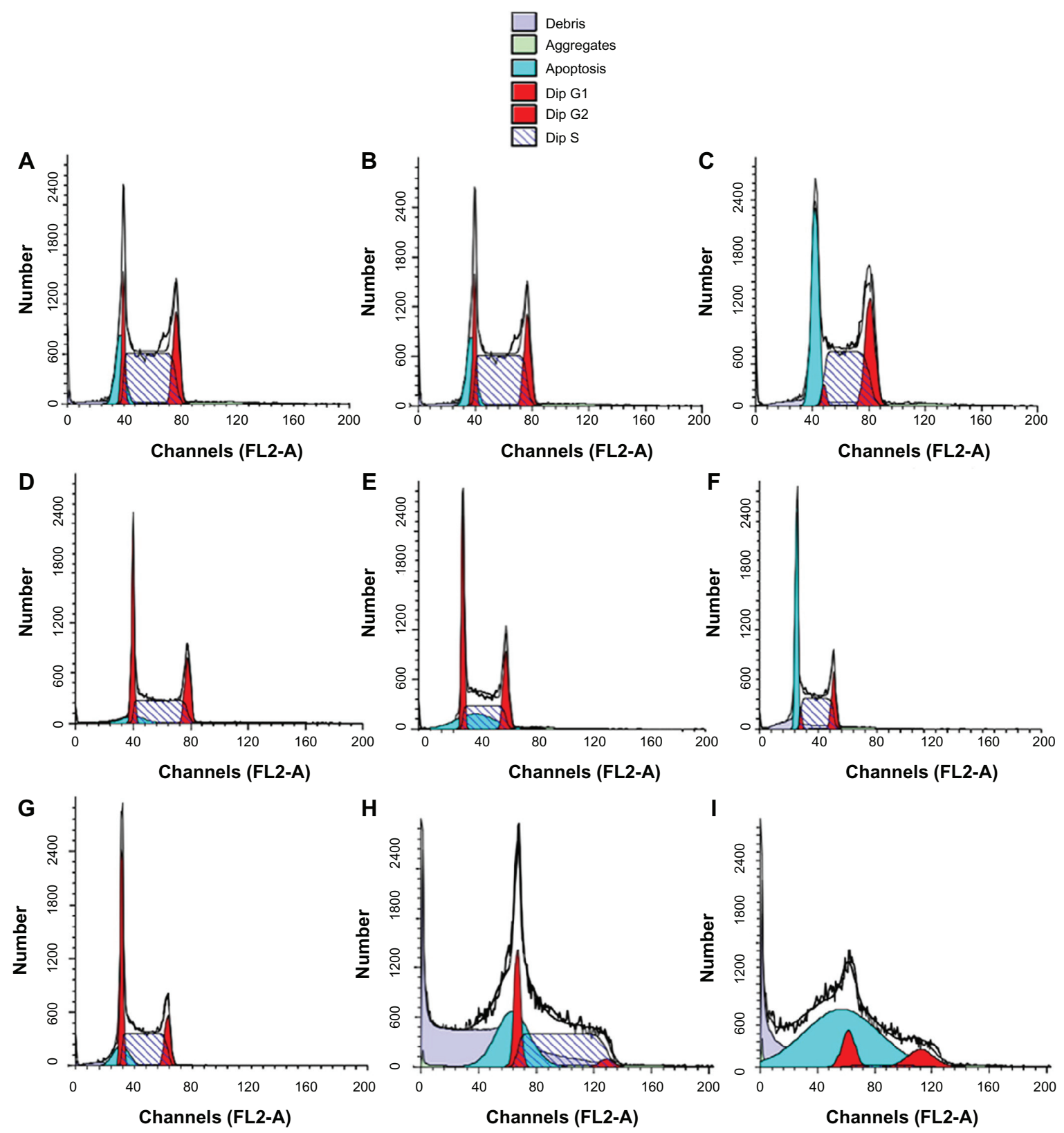

Figure 7 Flow cytometry cell cycle analysis of INA-6 cells. Control cells (A, D, and $\mathbf{G})$, cells treated with free gemcitabine (B, E, and $\mathbf{H})$, or with liposome-entrapped drug (C, F, and I) for 24 hours (A-C), 48 hours (D-F), or 72 hours (G-I). C) 2008, Elsevier. Reproduced with permission from Celia C, Malara N, Terracciano R et al. Liposomal delivery improves the growth-inhibitory and apoptotic activity of low doses of gemcitabine in multiple myeloma cancer cells. Nanomedicine. 2008;4(2): 155-166. ${ }^{55}$

Notes: The symbol representing cells in S-phase is indicated; the symbol representing cells in sub-GI phase is indicated as "apoptosis" because cells in the sub-GI phase are recognized as being apoptotic.

after inoculation of the colloidal formulation, while in the case of the free form, these were reached after 30 minutes, demonstrating the need for the active compound to be leaked from the carrier in order for it to be metabolized. ${ }^{11}$

To confirm the superior antitumoral activity of gemcitabine-loaded PEGylated liposomes with respect to free gemcitabine, the aforementioned formulation was compared with the commercial product, Gemzar ${ }^{\circledR}$, using in vivo models of anaplastic thyroid carcinoma in NOD-SCID mice bearing human anaplastic thyroid xenograft tumors. After 4 weeks of treatment, the antitumor activity of the colloidal formulation was similar to that of Gemzar at a drug dose which was ten times higher $(5 \mathrm{mg} / \mathrm{kg}$ of liposomal gemcitabine versus $50 \mathrm{mg} / \mathrm{kg}$ of the commercial form), in terms of average tumor size and volume. Furthermore, histological analysis of the tumors showed that there was no difference in organization of the neoplastic tissue..$^{50}$ Use of a lower drug concentration than that in the commercial form represents 


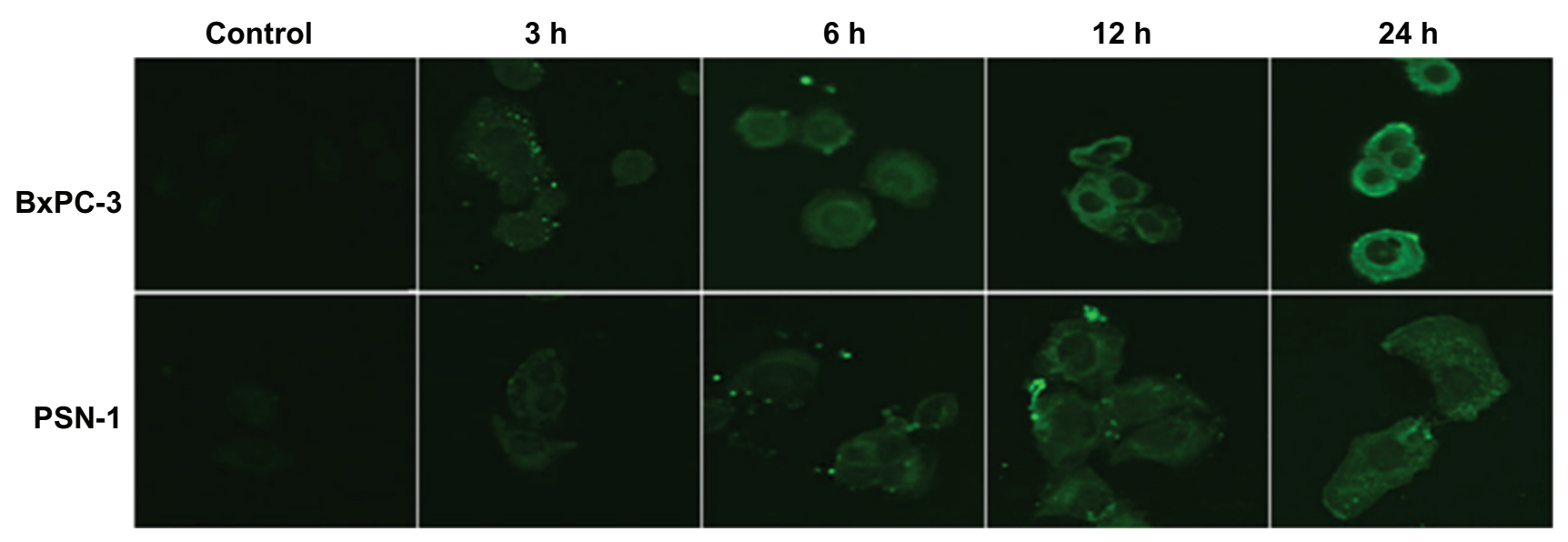

Figure 8 Interactions between gemcitabine-loaded PEGylated liposomes and pancreatic cancer cells. @ 20I I, Elsevier. Reproduced with permission from Yang F, Jin C, Jiang Y, et al. Liposome based delivery systems in pancreatic cancer treatment: from bench to bedside. Cancer Treat Rev. 201 I;37(8):633-642. ${ }^{25}$

Notes: Confocal laser scanning microscopy shows efficient interaction between gemcitabine-loaded PEGylated liposomes and BxPC-3 and PSN-I cell membranes. Intracellular localization of fluorescein-dihexadecanoyl phosphoethanolamine is time-dependent.

Abbreviation: PEG, poly(ethylene glycol).

a fundamental advance in anticancer therapy in terms of a possible reduction of side effects, particularly hematological toxicity. On the other hand, it should be pointed out that the absence of side effects was probably a consequence of both the low drug dose used and the treatment period which was too short to have any relevant toxicity. ${ }^{19}$

Another liposomal formulation, ie, vesicular phospholipid gel made up of hydrogenated egg phosphatidylcholine and cholesterol (in a molar ratio of 55:45), was tested in a human pancreatic ductal carcinoma cell line (MIA PaCa-2) grown in an orthotopic manner in nude mice. In this study, the antitumoral and antimetastatic activity of free and vesicular phospholipid gel-encapsulated gemcitabine were compared following intravenous administration using bioluminescence mediated by stable integration of the luciferase gene and in vivo imaging (Figure 9). The experiments used two different doses of liposomal gemcitabine ( $4 \mathrm{mg} / \mathrm{kg}$ and $8 \mathrm{mg} / \mathrm{kg}$ ), free gemcitabine $(240 \mathrm{mg} / \mathrm{kg})$, or the empty vehicle (equivalent to $8 \mathrm{mg} / \mathrm{kg}$ of liposomal gemcitabine) injected intravenously once weekly for 5 weeks. The in vivo bioluminescence results showed that liposomal gemcitabine (at a drug concentration of $8 \mathrm{mg} / \mathrm{kg}$ ) led to a reduction in tumor size by $68 \%$ with respect to the free drug which reduced tumor size by $7 \%$. Detection and quantification of the metastatic burden was performed visually and by luciferase assay of tissue obtained by resection and homogenization of potential target organs, including the pancreas, spleen, liver, stomach, intestine, lungs and local lymph nodes. Briefly, the animals were anesthetized, injected with $2 \mathrm{mg}$ of luciferin and, 10 minutes later, the light emitted by the tumors was collected over
5 minutes using a charge-coupled device camera. To validate the luciferase assays on tissue homogenates, frozen sections were prepared from the liver tissue of tumor-bearing animals, and luciferin and ATP were used to identify tumor cells in the sections. The emitted light was detected as mentioned, and the sections were subsequently stained with hematoxylin and eosin in order to analyze the morphology of areas with positive scores. Measurement of luciferase activity in the tissue homogenates provided a sensitive tool for analyzing and quantifying metastasizing tumor cells in the organs and to detect and quantify smaller metastases and those that were hidden internally. ${ }^{56}$ All formulations, including the empty liposomes, attenuated the spread of metastases. This investigation showed that luciferase-marked tumor cells are powerful tools and greatly aid the observation of in vivo tumor growth in addition to being able to detect and quantify metastases. ${ }^{56}$

Another study assessed the anticancer and antimetastatic activity of gemcitabine-loaded vesicular phospholipid gel in an orthotopic luciferase/green fluorescent protein-expressing LNCaP prostate cancer xenograft model in SCID mice. ${ }^{57}$ The results showed that liposomal gemcitabine decreased growth of the primary tumor and its metastatic diffusion, representing a possible treatment alternative for prostate cancer. Tumor growth was monitored once weekly by in vivo luciferase bioluminescence imaging of photons per second using the NightOwl LB981 charge-coupled device camera system (Berthold, Bad-Wildbach, Germany). The mice were treated with vesicular phospholipid gel gemcitabine $(8 \mathrm{mg} / \mathrm{kg})$ or the free form $(360 \mathrm{mg} / \mathrm{kg})$, with saline solution used as 


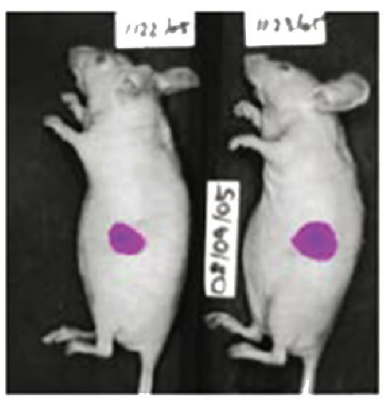

Day 7

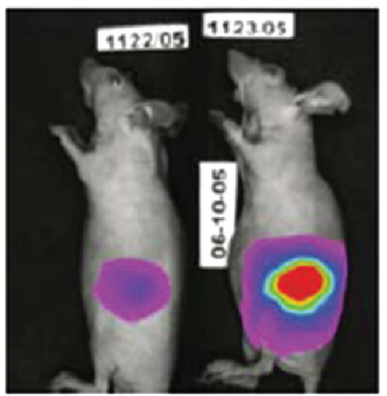

Day 35

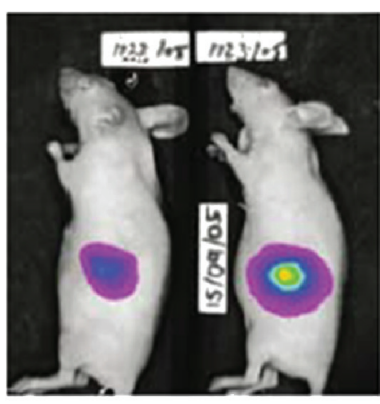

Day 14

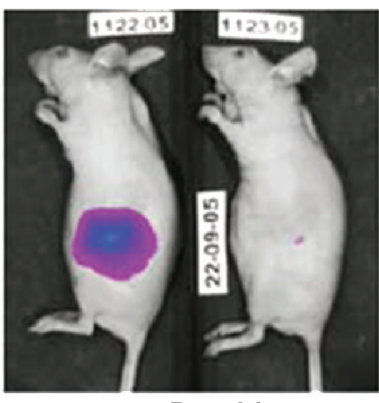

Day 21

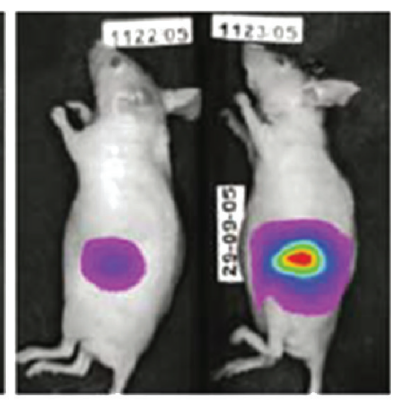

Day 28

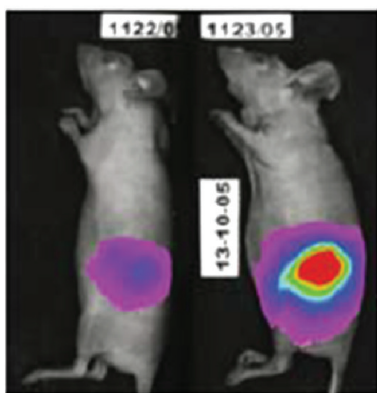

Day 42

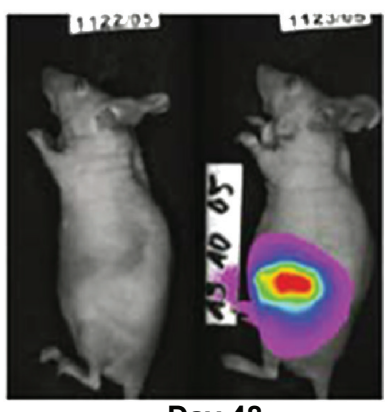

Day 48

Figure 9 Examples of in vivo luciferase measurements, quantification, and generation of tumor growth curves, showing one animal from the control group (right) and one from the liposomal gemcitabine $8 \mathrm{mg} / \mathrm{kg}$ group (left). () 2007, Springer. Reproduced with permission from Bornmann C, Graeser R, Esser N, et al. A new liposomal formulation of gemcitabine is active in an orthotopic mouse model of pancreatic cancer accessible to bioluminescence imaging. Cancer Chemother Pharmacol. 2008;6I(3):395-405. ${ }^{56}$ Notes: Overlays of a picture with the light signal encoded as a spectrum with red representing the most, and blue the least intense light, are shown. Tumor end-volumes were $0.45 \mathrm{~cm}^{3}(1,122)$, and $1.69 \mathrm{~cm}^{3}(1,123)$, respectively.

the control. The resulting data showed a $83.9 \%$ decrease in tumor growth in mice with gemcitabine in the vesicular phospholipid gel and a $70.9 \%$ decrease using the free form of the drug, as well as a drop in metastatic spread, eg, to the lymph nodes, lungs, kidneys, and stomach. ${ }^{57}$ Unfortunately, vesicular phospholipid gel liposomes are very viscous and cannot be administered in vivo in this gel-like form and need to be diluted before intravenous administration, thus inducing rapid drug leakage and substantial pharmacokinetic fluctuation. ${ }^{58}$

A further strategy is based on conjugation of targeting moieties, such as the epidermal growth factor receptor antibody in PEG chains surrounding the surfaces of $\mathrm{pH}-$ sensitive liposomes. This technique enhanced the antitumoral activity of gemcitabine in A549 non-small lung cancer cells and their xenografts in mouse models with respect to the PEGylated liposomal formulation. ${ }^{59}$ Further investigation showed that effective in vivo delivery of gemcitabine by the liposomes is attributable to the smaller mean size of the vesicles, coating of their surfaces with biocompatible hydrophilic molecules, high drug-loading values inside the carriers, and the opportunity to bind suitable molecules on their surfaces in order to obtain selective targeting in specific tissues.

\section{Multidrug carriers}

In recent years, many research teams have investigated the anticancer efficacy of a single drug encapsulated in a PEGylated liposomal formulation, evaluating physicochemical and technological characteristics as well as potential side effects in in vitro and in vivo studies. ${ }^{60}$ As previously described, colloidal vesicles can attenuate the biopharmaceutical properties of bioactive agents, allowing an increase in their pharmacological effects as well as modulation of their pharmacokinetic parameters (eg, plasma half-life) as a consequence of being protected from metabolic pathways. ${ }^{18,61}$ For example, encapsulation of the anthracycline, doxorubicin, approved and commercialized as Doxil and Caelyx, reduced its side effects, in particular its cardiotoxicity and myelotoxicity. ${ }^{62,63}$

In view of these observations, further experimentation was undertaken based on the synergistic action of two or more anticancer drugs coencapsulated in the same colloidal device, with the aim of finding a starting point for the development of liposomal multidrug carriers. The concept of multidrug carriers is an innovative strategy in the fight against cancer, because it could serve as a springboard for reduction of the effective drug dose, thereby reducing the side effects of traditional therapies. The rationale for this approach is closely related to the possibility of acting upon different steps in the 
same cascade of events responsible for the transformation of a healthy cell into a malignant one. ${ }^{64}$

For example, Tardi et al demonstrated that the simultaneous presence of two water-soluble compounds, irinotecan and floxuridine, in a liposomal device did not destabilize the vesicular structure, and a synergistic effect of the drugs was confirmed in in vitro and in vivo studies, along with modulation of their biopharmaceutical and pharmacokinetic profiles. ${ }^{65,66}$ Similar results were obtained in another study involving coencapsulation of irinotecan and cisplatin in a liposomal carrier which demonstrated increased antitumoral efficacy in the treatment of small cell lung cancer. ${ }^{67}$

A new multidrug carrier containing a hydrophilic drug (located within the aqueous liposomal compartments) and a lipophilic compound (encapsulated in the bilayers), ie, gemcitabine and paclitaxel respectively, was recently developed. ${ }^{68}$ As previously reported, gemcitabine induces $\mathrm{G}_{0} / \mathrm{G}_{1}$-phase and S-phase arrest and triggers apoptosis in solid tumor cells, ${ }^{6}$ whereas paclitaxel acts as a mitotic spindle poison by blocking eukaryotic cells in the $G_{2} / M$ mitotic phase of the cell cycle, thereby triggering apoptotic cell death. Furthermore, considering their partially nonoverlapping toxicities, the aim of coencapsulating these agents within a liposomal device was to reduce both the effective drug dose and the side effects of both active compounds.

The association of gemcitabine and paclitaxel in the same colloidal device did not induce significant variations in mean particle size as compared with the empty liposomes or liposomes containing either single drug alone. Analysis of parameters such as polydispersity index and zeta potential confirmed that the presence of either drug alone allowed the liposomal formulations to maintain their physicochemical features, but the simultaneous presence of both antitumoral compounds induced a slight decrease in encapsulation values.

Use of multidrug carriers for breast cancer (MCF-7) cells had good antitumoral activity in vitro, confirming that the multidrug carrier formulation is more cytotoxic, considering the drug concentration and exposure time, with respect to the free compounds alone or their respective liposomal formulations. Flow cytometry showed synergistic in vitro antitumoral action between the two antitumoral compounds coencapsulated in the same device. Treatment with the multidrug carrier induced predominantly $\mathrm{G}_{2}$ cell arrest, with a reappearance of $\mathrm{G}_{0} / \mathrm{G}_{1}$ and $\mathrm{S}$ phase processes as a consequence of the combined anticancer action of the two drugs. ${ }^{68}$
Recently, a novel PEGylated liposomal multidrug carrier encapsulating gemcitabine and tamoxifen, a nonsteroidal selective estrogen receptor modulator, was devised with the aim of developing a new nanomedicine for the treatment of breast cancer. ${ }^{69}$ Tamoxifen is an ideal synergistic agent able to increase the antineoplastic effect of nucleoside analogs. ${ }^{70,71}$ The antitumoral activity of the multidrug carrier was tested in two human breast cancer (MCF-7 and T47D) cell lines and compared with the single drugs administered both in free form and as liposomal formulations. Treatment with a combination of the two drugs, and especially with the multidrug carrier, resulted in significant reduction of cell viability after 24 hours of incubation in both cell lines with

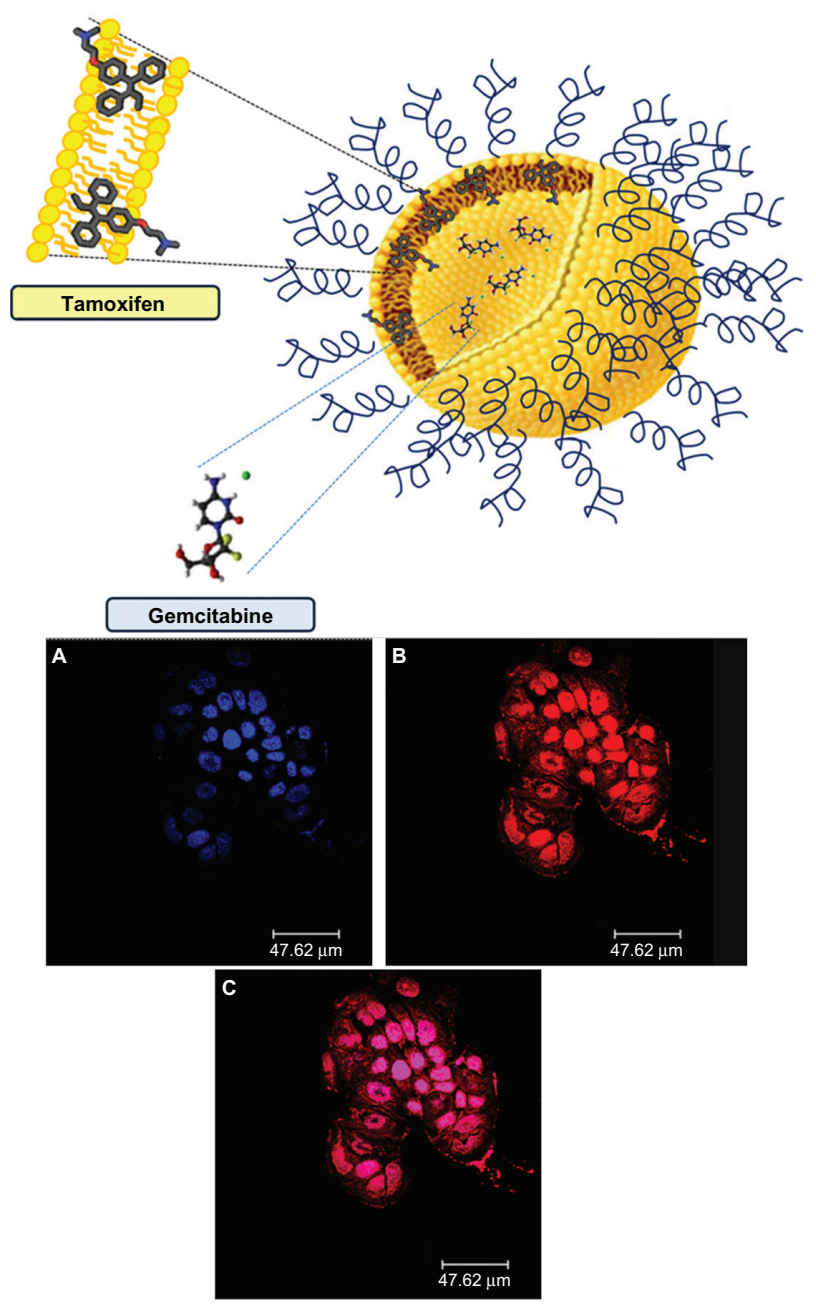

Figure 10 Schematic representation of gemcitabine-tamoxifen localization inside the multidrug carriers (upper panel). Confocal laser scanning micrographs of T47D cells treated with rhodamine-labeled PEGylated unilamellar liposomes after 6 hours of incubation. (A) Hoechst filter, (B) TRITC filter, and (C) overlay (lower panel). Note: (C) 2012, Elsevier. Reproduced with permission from Cosco D, Paolino D, Cilurzo F, Casale F, Fresta M. Gemcitabine and tamoxifen-loaded liposomes as multidrug carriers for the treatment of breast cancer diseases. Int J Pharm. 2012; 422(I-2):229-237.69

Abbreviation: PEG, poly(ethylene glycol). 
respect to the lowest drug dose tested in its free form. This trend was even more evident after 48 and 72 hours in terms of the incubation time and drug concentration used. This was probably due to the synergistic action of the coencapsulated drugs favored by the colloidal device which promoted their cellular internalization and accumulation, thus avoiding destabilization phenomena. Further evidence has been gathered from cell-carrier interaction studies using CLSM, eg, after 6 hours of incubation with rhodamine-labeled liposomes, it was possible to detect red staining of all cellular microenvironments (Figure 10). ${ }^{69}$

\section{Conclusion}

The use of innovative colloidal delivery systems represents a valid option in cancer therapy. The possibility of reducing or overcoming the side effects related to administration of anticancer drugs in their free form and especially the option of coencapsulating two drugs having different physicochemical properties and different mechanisms of action in the same carrier represents an ambitious challenge for modern technology. In particular, combination therapy and the concept of novel nanodevices which are more selective for tumoral cells are very promising research fields in the treatment of cancer. Moreover, the concept of novel polymers and copolymers able to act as masking systems and offering the opportunity to conjugate different molecules could represent a further advance in the selective targeting of gemcitabine against tumors. $^{72,73}$

\section{Acknowledgment}

The authors are very grateful to Lynn Whitted for her revision of the manuscript.

\section{Disclosure}

The authors report no conflicts of interest in this work.

\section{References}

1. Cavallaro G, Licciardi M, Salmaso S, Caliceti P, Giammona G. Folatemediated targeting of polymeric conjugates of gemcitabine. Int J Pharm. 2006;307(2):258-269.

2. Immordino ML, Dosio F, Cattel L. Stealth liposomes: review of the basic science, rationale, and clinical applications, existing and potential. Int $J$ Nanomedicine. 2006;1(3):297-315.

3. Trapani G, Denora N, Trapani A, Laquintana V. Recent advances in ligand targeted therapy. J Drug Target. 2012;20(1):1-22.

4. Heinemann V. Role of gemcitabine in the treatment of advanced and metastatic breast cancer. Oncology. 2003;64(3):191-206.

5. Pauwels B, Korst AE, Lardon F, Vermorken JB. Combined modality therapy of gemcitabine and radiation. Oncologist. 2005;10(1):34-51.

6. Mini E, Nobili S, Caciagli B, Landini I, Mazzei T. Cellular pharmacology of gemcitabine. Ann Oncol. 2006;17 Suppl 5:7-12.
7. Lostao MP, Mata JF, Larrayoz IM, et al. Electrogenic uptake of nucleosides and nucleoside-derived drugs by the human nucleoside transporter 1 (hCNT1) expressed in Xenopus laevis oocytes. FEBS Lett. 2000;481(2):137-140.

8. Ueno H, Kiyosawa K, Kaniwa N. Pharmacogenomics of gemcitabine: can genetic studies lead to tailor-made therapy? Br J Cancer. 2007;97(2):145-151.

9. Ritzel MW, Ng AM, Yao SY, et al. Recent molecular advances in studies of the concentrative-dependent nucleoside transporter (CNT) family: identification and characterization of novel human and mouse proteins (hCNT3 and mCNT3) broadly selective for purine and pyrimidine nucleosides (system cib). Mol Membr Biol. 2001;18(1): 65-72.

10. Bhutia YD, Hung SW, Patel B, Lovin D, Govindarajan R. CNT1 expression influences proliferation and chemosensitivity in drug-resistant pancreatic cancer cells. Cancer Res. 2011;71(5):1825-1835.

11. Cosco D, Bulotta A, Ventura M, et al. In vivo activity of gemcitabineloaded PEGylated small unilamellar liposomes against pancreatic cancer. Cancer Chemother Pharmacol. 2009;64(5):1009-1020.

12. Allen C, Dos SN, Gallagher R, et al. Controlling the physical behavior and biological performance of liposome formulations through use of surface grafted poly(ethylene glycol). Biosci Rep. 2002;22(2): 225-250.

13. Knop K, Hoogenboom R, Fischer D, Schubert US. Poly(ethylene glycol) in drug delivery: pros and cons as well as potential alternatives. Angew Chem Int Ed Engl. 2010;49(36):6288-6308.

14. Bergman AM, Pinedo HM, Peters GJ. Determinants of resistance to $2^{\prime}, 2^{\prime}$-difluorodeoxycytidine (gemcitabine). Drug Resist Updat. 2002;5(1):19-33.

15. Brusa P, Immordino ML, Rocco F, Cattel L. Antitumor activity and pharmacokinetics of liposomes containing lipophilic gemcitabine prodrugs. Anticancer Res. 2007;27(1A):195-199.

16. Ohhashi S, Ohuchida K, Mizumoto K, et al. Down-regulation of deoxycytidine kinase enhances acquired resistance to gemcitabine in pancreatic cancer. Anticancer Res. 2008;28(4B):2205-2212.

17. Zhang X, Yashiro M, Qiu H, et al. Establishment and characterization of multidrug-resistant gastric cancer cell lines. Anticancer Res. 2010;30(3):915-921.

18. Gabizon AA, Shmeeda H, Zalipsky S. Pros and cons of the liposome platform in cancer drug targeting. J Liposome Res. 2006;16(3):175-183.

19. Dasanu CA. Gemcitabine: vascular toxicity and prothrombotic potential. Expert Opin Drug Saf. 2008;7(6):703-706.

20. Reddy LH, Couvreur P. Novel approaches to deliver gemcitabine to cancers. Curr Pharm Des. 2008;14(11):1124-1137.

21. Celia C, Cosco D, Paolino D, Fresta M. Gemcitabine-loaded innovative nanocarriers vs Gemzar: Biodistribution, pharmacokinetic features and in vivo antitumor activity. Expert Opin Drug Deliv. 2011;8(12):1609-1629.

22. Barenholz YC. Doxil ${ }^{\circledR}$ - The first FDA-approved nano-drug: lessons learned. J Control Release. 2012;160(2):117-134.

23. Paolino D, Cosco D, Cilurzo F, Fresta M. Innovative drug delivery systems for the administration of natural compounds. Curr Bioact Compd. 2007;3:262-277.

24. Muthu MS, Singh S. Targeted nanomedicines: effective treatment modalities for cancer, AIDS and brain disorders. Nanomedicine (Lond). 2009;4(1):105-118.

25. Yang F, Jin C, Jiang Y, et al. Liposome based delivery systems in pancreatic cancer treatment: from bench to bedside. Cancer Treat Rev. 2011;37(8):633-642.

26. Torchilin V. Antibody-modified liposomes for cancer chemotherapy. Expert Opin Drug Del. 2008;5(9):1003-1025.

27. Yan X, Scherphof GL, Kamps JA. Liposome opsonization. J Liposome Res. 2005;15(1-2):109-139.

28. Decuzzi P, Causa F, Ferrari M, Netti PA. The effective dispersion of nanovectors within the tumor microvasculature. Ann Biomed Eng. 2006;34(4):633-641. 
29. Cosco D, Paolino D, Muzzalupo R, et al. Novel PEG-coated niosomes based on bola-surfactant as drug carriers for 5-fluorouracil. Biomed Microdevices. 2009;11(5):1115-1125.

30. Pasut G, Veronese FM. PEGylation for improving the effectiveness of therapeutic biomolecules. Drugs Today (Barc). 2009;45(9): 687-695.

31. Veronese FM, Pasut G. PEGylation, successful approach to drug delivery. Drug Discov Today. 2005;10(21):1451-1458.

32. Gabizon A, Shmeeda H, Barenholz Y. Pharmacokinetics of pegylated liposomal doxorubicin: review of animal and human studies. Clin Pharmacokinet. 2003;42(5):419-436.

33. Niu G, Cogburn B, Hughes J. Preparation and characterization of doxorubicin liposomes. Methods Mol Biol. 2010;624:211-219.

34. Duggan ST, Keating GM. Pegylated liposomal doxorubicin: a review of its use in metastatic breast cancer, ovarian cancer, multiple myeloma and AIDS-related Kaposi's sarcoma. Drugs. 2011;71(18): 2531-2558.

35. Celano M, Calvagno MG, Bulotta S, et al. Cytotoxic effects of gemcitabine-loaded liposomes in human anaplastic thyroid carcinoma cells. BMC Cancer. 2004;4:63.

36. Calvagno MG, Celia C, Paolino D, et al. Effects of lipid composition and preparation conditions on physical-chemical properties, technological parameters and in vitro biological activity of gemcitabine-loaded liposomes. Curr Drug Deliv. 2007;4(1):89-101.

37. Castelli F, Raudino A, Fresta M. A mechanistic study of the permeation kinetics through biomembrane models: gemcitabinephospholipid bilayer interaction. J Colloid Interface Sci. 2005;285(1): 110-117.

38. Liang W, Levchenko T, Khaw BA, Torchilin V. ATP-containing immunoliposomes specific for cardiac myosin. Curr Drug Deliv. 2004;1(1):1-7.

39. Immordino ML, Brusa P, Rocco F, Arpicco S, Ceruti M, Cattel L. Preparation, characterization, cytotoxicity and pharmacokinetics of liposomes containing lipophilic gemcitabine prodrugs. J Control Release. 2004;100(3):331-346.

40. Couvreur P, Stella B, Reddy LH, et al. Squalenoyl nanomedicines as potential therapeutics. Nano Lett. 2006;6(11):2544-2548.

41. Reddy LH, Dubernet C, Mouelhi SL, Marque PE, Desmaele D, Couvreur P. A new nanomedicine of gemcitabine displays enhanced anticancer activity in sensitive and resistant leukemia types. J Control Release. 2007;124(1-2):20-27.

42. Pili B, Reddy LH, Bourgaux C, Lepetre-Mouelhi S, Desmaele D, Couvreur P. Liposomal squalenoyl-gemcitabine: formulation, characterization and anticancer activity evaluation. Nanoscale. 2010;2(8):1521-1526.

43. Ishida T, Harada M, Wang XY, Ichihara M, Irimura K, Kiwada H. Accelerated blood clearance of PEGylated liposomes following preceding liposome injection: effects of lipid dose and PEG surfacedensity and chain length of the first-dose liposomes. J Control Release. 2005;105(3):305-317.

44. Dams ET, Laverman P, Oyen WJ, et al. Accelerated blood clearance and altered biodistribution of repeated injections of sterically stabilized liposomes. J Pharmacol Exp Ther. 2000;292(3):1071-1079.

45. Ishida T, Kiwada H. Accelerated blood clearance (ABC) phenomenon upon repeated injection of PEGylated liposomes. Int J Pharm. 2008;354(1-2):56-62.

46. Paolino D, Cosco D, Molinaro R, Celia C, Fresta M. Supramolecular devices to improve the treatment of brain diseases. Drug Discov Today. 2011;16(7-8):311-324.

47. Ishihara T, Maeda T, Sakamoto H, et al. Evasion of the accelerated blood clearance phenomenon by coating of nanoparticles with various hydrophilic polymers. Biomacromolecules. 2010;11(10): 2700-2706.

48. Xu H, Wang KQ, Deng YH, Chen da W. Effects of cleavable PEGcholesterol derivatives on the accelerated blood clearance of PEGylated liposomes. Biomaterials. 2010;31(17):4757-4763.
49. Pasut G, Canal F, Dalla Via L, Arpicco S, Veronese FM, Schiavon O. Antitumoral activity of PEG-gemcitabine prodrugs targeted by folic acid. J Control Release. 2008;127(3):239-248.

50. Paolino D, Cosco D, Racanicchi L, et al. Gemcitabine-loaded PEGylated unilamellar liposomes vs GEMZAR: biodistribution, pharmacokinetic features and in vivo antitumor activity. $J$ Control Release. 2010;144(2):144-150.

51. Vono M, Cosco D, Celia C, et al. In vitro evaluation of the activity of gemcitabine-loaded pegylated unilamellar liposomes against papillary thyroid cancer cells. The Open Drug Delivery Journal. 2010;4:55-62.

52. Dan N. Effect of liposome charge and PEG polymer layer thickness on cell-liposome electrostatic interactions. Biochim Biophys Acta. 2002;1564(2):343-348.

53. Borowik T, Widerak K, Ugorski M, Langner M. Combined effect of surface electrostatic charge and poly(ethyl glycol) on the association of liposomes with colon carcinoma cells. J Liposome Res. 2005;15(3-4):199-213.

54. Celia C, Calvagno MG, Paolino D, et al. Improved in vitro anti-tumoral activity, intracellular uptake and apoptotic induction of gemcitabineloaded pegylated unilamellar liposomes. J Nanosci Nanotechnol. 2008;8(4):2102-2113

55. Celia C, Malara N, Terracciano R, et al. Liposomal delivery improves the growth-inhibitory and apoptotic activity of low doses of gemcitabine in multiple myeloma cancer cells. Nanomedicine. 2008;4(2):155-166.

56. Bornmann C, Graeser R, Esser N, et al. A new liposomal formulation of gemcitabine is active in an orthotopic mouse model of pancreatic cancer accessible to bioluminescence imaging. Cancer Chemother Pharmacol. 2008;61(3):395-405.

57. Jantscheff P, Ziroli V, Esser N, et al. Anti-metastatic effects of liposomal gemcitabine in a human orthotopic LNCaP prostate cancer xenograft model. Clin Exp Metastasis. 2009;26(8):981-992.

58. Moog R, Burger AM, Brandl M, et al. Change in pharmacokinetic and pharmacodynamic behavior of gemcitabine in human tumor xenografts upon entrapment in vesicular phospholipid gels. Cancer Chemother Pharmacol. 2002;49(5):356-366.

59. Kim IY, Kang YS, Lee DS, et al. Antitumor activity of EGFR targeted $\mathrm{pH}$-sensitive immunoliposomes encapsulating gemcitabine in A549 xenograft nude mice. J Control Release. 2009;140(1):55-60.

60. Celano M, Schenone S, Cosco D, et al. Cytotoxic effects of a novel pyrazolopyrimidine derivative entrapped in liposomes in anaplastic thyroid cancer cells in vitro and in xenograft tumors in vivo. Endocr Relat Cancer. 2008;15(2):499-510.

61. Drulis-Kawa Z, Dorotkiewicz-Jach A. Liposomes as delivery systems for antibiotics. Int J Pharm. 2010;387(1-2):187-198.

62. Robert NJ, Vogel CL, Henderson IC, et al. The role of the liposomal anthracyclines and other systemic therapies in the management of advanced breast cancer. Semin Oncol. 2004;31(6 Suppl 13):106-146.

63. Injac R, Strukelj B. Recent advances in protection against doxorubicininduced toxicity. Technol Cancer Res Treat. 2008;7(6):497-516.

64. Colomer R. What is the best schedule for administration of gemcitabinetaxane? Cancer Treat Rev. 2005;31 Suppl 4:S23-S28.

65. Harasym TO, Tardi PG, Harasym NL, Harvie P, Johnstone SA, Mayer LD. Increased preclinical efficacy of irinotecan and floxuridine coencapsulated inside liposomes is associated with tumor delivery of synergistic drug ratios. Oncol Res. 2007;16(8):361-374.

66. Tardi PG, Gallagher RC, Johnstone S, et al. Coencapsulation of irinotecan and floxuridine into low cholesterol-containing liposomes that coordinate drug release in vivo. Biochim Biophys Acta. 2007;1768(3):678-687.

67. Tardi PG, Dos Santos N, Harasym TO, et al. Drug ratio-dependent antitumor activity of irinotecan and cisplatin combinations in vitro and in vivo. Mol Cancer Ther. 2009;8(8):2266-2275.

68. Cosco D, Paolino D, Maiuolo J, Russo D, Fresta M. Liposomes as multicompartmental carriers for multidrug delivery in anticancer chemotherapy. Drug Deliv Transl Res. 2011;1:66-75. 
69. Cosco D, Paolino D, Cilurzo F, Casale F, Fresta M. Gemcitabine and tamoxifen-loaded liposomes as multidrug carriers for the treatment of breast cancer diseases. Int J Pharm. 2012;422(1-2):229-237.

70. Zheng A, Kallio A, Härkönen P. Tamoxifen-induced rapid death of MCF-7 breast cancer cells is mediated via extracellularly signalregulated kinase signaling and can be abrogated by estrogen. Endocrinology. 2007;148(6):2764-2777.

71. Brauch H, Jordan VC. Targeting of tamoxifen to enhance antitumor action for the treatment and prevention of breast cancer: the 'personalised' approach? Eur J Cancer. 2009;45(13):2274-2283.
72. Paolino D, Cosco D, Licciardi M, Giammona G, Fresta M, Cavallaro G. Polyaspartyl hydrazide copolymer-based supramolecular vesicular aggregates as delivery devices for anticancer drugs. Biomacromolecules. 2008;9(4):1117-1130.

73. Licciardi M, Paolino D, Celia C, Giammona G, Cavallaro G, Fresta M. Folate-targeted supramolecular vesicular aggregates based on polyaspartyl-hydrazide copolymers for the selective delivery of antitumoral drugs. Biomaterials. 2010;31(28):7340-7354.

\section{Publish your work in this journal}

The International Journal of Nanomedicine is an international, peerreviewed journal focusing on the application of nanotechnology in diagnostics, therapeutics, and drug delivery systems throughout the biomedical field. This journal is indexed on PubMed Central, MedLine, CAS, SciSearch ${ }^{\circledR}$, Current Contents ${ }^{\circledR} /$ Clinical Medicine,
Journal Citation Reports/Science Edition, EMBase, Scopus and the Elsevier Bibliographic databases. The manuscript management system is completely online and includes a very quick and fair peer-review system, which is all easy to use. Visit http://www.dovepress.com/ testimonials.php to read real quotes from published authors. 